\title{
Incidence and microbiology of post- operative infections after radical cystectomy and ureteral stent removal; a retrospective cohort study
}

E. Kolwijck ${ }^{1,2^{*}}$, A. E. M. Seegers ${ }^{1,2,3}$, S. C. M. Tops ${ }^{1,2,3}$, A. G. van der Heijden ${ }^{4}$, J. P. M. Sedelaar ${ }^{4}$ and J. ten Oever ${ }^{1,3}$

\begin{abstract}
Background: Post-operative infections are frequent after radical cystectomy with urinary diversion surgery (UDS). Reduction of post-operative infections necessitates appropriate peri-operative antimicrobial prophylaxis targeting causative bacteria. We assessed the incidence and microbiology of infections in the 30-day post-operative period after UDS and investigated effectiveness of the currently used peri-operative antibacterial prophylaxis.

Methods: Retrospective cohort study of all patients undergoing UDS in a tertiary university medical center from January 2014 until September 2016. Antibiotic prophylaxis consisted of cefazolin plus metronidazol according to the Dutch national guideline. Primary outcome was the incidence of post-operative infections within 30 days. Risk factors for post-operative infections and antimicrobial susceptibility profiles of cultured bacteria were also assessed.

Results: 147 patients were included. 69 patients (46.9\%) had 82 post-operative infections, 27 of which were patients with bacteremia (18.4\%). Highest incidence of infections was on day 4-5 and on day 8-10 postoperatively. The second peak was associated with ureteral stent removal. $4.8 \%$ of 147 study patients developed bacteremia $24 \mathrm{~h}$ after stent removal, which counted for $25.9 \%$ of all bacteremia episodes found in this study. Enterobacteriaceae were cultured in $67.9 \%$ of blood cultures and were only highly susceptible to ciprofloxacine, piperacillin-tazobactam (90\%), meropenem and gentamicin (100\%). Multivariate logistic regression analysis showed orthotopic Hautmann neobladder to be associated with increased infections complications: odds ratio 4.1 (95\% confidence interval 1.6-10.5), $p=0.03$.

Conclusions: The incidence of infections after radical cystectomy is high and particularly ureteral stent removal was associated with both bacteremia and complicated urinary tract infections. Based on the results of this study, antibiotic prophylaxis might need to be broadened for patients undergoing radical cystectomy. Further research is required to investigate whether current guidelines need to be altered concerning administration of antibiotic prophylaxis just before stent removal.
\end{abstract}

Keywords: Antibiotic prophylaxis, Post-operative infection, Ureteral stent, Radical cystectomy, Microbiology

\footnotetext{
* Correspondence: eva.kolwijck@radboudumc.nl;

eva.kolwijck@radboudumc.nl

${ }^{1}$ Radboud center for infectious diseases, Radboud university medical center,

P.O. Box 9101, 6500, HB, Nijmegen, the Netherlands

${ }^{2}$ Department of medical microbiology, Radboud university medical center,

P.O. Box 9101, 6500, HB, Nijmegen, the Netherlands

Full list of author information is available at the end of the article
}

(c) The Author(s). 2019 Open Access This article is distributed under the terms of the Creative Commons Attribution 4.0 International License (http://creativecommons.org/licenses/by/4.0/), which permits unrestricted use, distribution, and

reproduction in any medium, provided you give appropriate credit to the original author(s) and the source, provide a link to the Creative Commons license, and indicate if changes were made. The Creative Commons Public Domain Dedication waiver (http://creativecommons.org/publicdomain/zero/1.0/) applies to the data made available in this article, unless otherwise stated. 


\section{Background}

Ileal conduit (IC) and orthotopic Hautmann Neobladder (NB) are two types of urinary diversion surgery (UDS) that are frequently performed after radical cystectomy. In both types of UDS, the ureters are attached to the IC or NB using temporal ureteral stents to assure patency and prevent stenosis while healing. The UDS procedure is associated with increased risk of bacteremia, complicated urinary tract infection (cUTI) and surgical site infection (SSI) [1]. The first 30 days, infections contribute significantly to post-operative morbidity $[2,3]$, which can be explained by multiple comorbidities as well as by the complex surgery that involves attaching part of the colonized gastro-intestinal tract to the sterile urinary tract [4].

Up until now, several studies focused on the incidence of early (<30 days) post-operative infections [4-11]. Overall infectious complication rates in these studies ranged from 10 to $41 \%[7,9,11]$. Of these infectious complications, bacteremia was reported in 5 to $17 \%$ of the patients [9-11], cUTI was reported in 4 to $36 \%$ of the patients [5, 9-11] and SSI was reported in 8 to $33 \%$ of patients [4, 6-9, 11] after UDS, even when peri-operative antimicrobial prophylaxis was used. Although some of these studies reported on the causative bacteria $[4,5,8-10]$, we found that only a few studies partially provided information on antimicrobial susceptibility of the cultured bacteria $[4,8,9]$. Particularly in the era of increasing antimicrobial resistance, susceptibility profiles provide an important pillar in the correct use of antimicrobial prophylaxis $[7,12]$. The recommended guideline-based peri-operative antimicrobial prophylaxis for UDS in the Netherlands consists of cefazolin plus metronidazol [13]. Cefazolin is one of the most prescribed (40\%) prophylactic antibiotics for radical cystectomy in the United States as well [14]. However, the same large cohort study showed that a penicillin-based regimen with a beta-lactamase inhibitor was associated with the least post-operative complications [14], suggesting increased gram-negative coverage is necessary in the era of increasing resistance.

Guidelines [13, 15] advise not to extend the prophylaxis beyond $24 \mathrm{~h}$ after surgery, which is further supported by a recent study [14]. Interestingly, none of the guidelines specifically recommends on the need and type of antibiotic prophylaxis during ureteral stent removal after UDS [15-17]. Ureteral stent removal is typically performed at least a week after UDS and involves manipulation in a non-sterile environment attached to the urological tract, which might predispose to infections, similar to manipulation of an obstructed common bile duct [18]. European [15] and American guidelines [16] do recommend antibiotic prophylaxis for patients undergoing cystourethroscopy (low quality of evidence) based on a few studies that assessed effectiveness of prophylactic antibiotics during upper urinary tract stone treatment, but this might not be representative to ureteral stent removal after UDS.

Given these shortcomings, the aim of this study was to ascertain the efficacy of antimicrobial prophylaxis by investigating the incidence and antibiotic susceptibility of causative micro-organisms of post-operative infections after IC or NB urinary diversion surgery. Additionally, we specifically investigated the incidence and microbiology of infections after ureteral stent removal.

\section{Methods}

We conducted a retrospective cohort study in the Radboud university medical center, a university hospital in the Netherlands performing $80-100$ radical cystectomies per year, by using a prospective database of patients undergoing radical cystectomy followed by UDS.

Inclusion criteria were patients aged $\geq 18$ years and undergoing either IC or NB diversion between January 2014 and September 2016 in the Radboud university medical center. Exclusion criteria were an active pre-operative infection, defined as the presence of signs of overt infection, or the prescription of a therapeutic course of antibiotics.

Preparation of patients undergoing UDS consisted of bowel preparation using an osmotic laxative and enemas starting one day prior to surgery. Antibiotic prophylaxis protocol according to the Dutch national guideline consisted of one dose of cefazolin $1000 \mathrm{mg}$ and metronidazole $500 \mathrm{mg}$ administered intravenously prior to incision [17]. In the Dutch guideline no antibiotic prophylaxis is recommended during ureteral stent removal, also no prolonged post-operative prophylaxis is recommended [17]. For a cefazolin dose to be scored as optimal guideline-adherent in our regression analyses, a repeat administration was required in case of prolonged surgery $(>4 \mathrm{~h})$, heavy blood loss $(>2 \mathrm{~L})$, and a double dose in case of a body mass index (BMI) $>35 \mathrm{~kg} / \mathrm{m}^{2}$ or bodyweight $>120 \mathrm{~kg}$ [19]. Removal of the ureteral stents was generally performed on two consecutive days, on day 7 and 8 in case of IC and on day 9 and 10 in case of NB after radical cystectomy.

Data of interest were extracted from the electronic medical record (EPIC) and entered in CastorEDC, a licensed online data collection directory for medical research. The following patient characteristics were collected: sex, age, length, weight, body mass index, American Society of Anesthesiologists (ASA) score, Charlson Comorbidity Index (CCI) score, indication for surgery and T-staging in the case of bladder cancer, previous chemotherapy, and carrier state of a multidrug resistant micro-organism (MDRO) (methicillin-resistant Staphylococcus aureus, vancomycin-resistant enterococci, bacteria containing plasmid-mediated AmpC beta-lactamases, extended-spectrum beta-lacamase producing Enterobacteriaceae, or carbapenemase-producing 
Enterobacteriaceae). Peroperative variables that were collected included duration of surgery, blood loss, timing and dosage of antibiotic prophylaxis and presence of nephrostomy or double-J catheters. Post-operative variables that were collected were (timing of) infections, length of stay and mortality. Table 1 shows the definitions used to classify infections.

The primary outcome measurement was the incidence of post-operative infections either microbiologically confirmed (bacteremia and cUTI) or clinically diagnosed (surgical site infection [SSI] [20]) within 30 days after radical cystectomy (Table 1). These infections were chosen since these can be prevented with preoperative antibiotic prophylaxis. A positive urine culture without concomitant bacteremia in a febrile patient shortly after UDS not necessarily indicates a cUTI, but excluding these patients would underestimate the incidence. Furthermore, the new urinary reservoir is the main portal of entry and the susceptibility of the residing bacteria should guide prophylaxis and empiric treatment. Patients with both a positive blood culture and a positive urine culture taken on the same day ( \pm 1 day) were scored as "bacteremia" and not as "cUTI". Secondary outcome measurements were timing of these infections with a specific interest in the relationship with ureteral stent removal, the susceptibility profiles of the causative microorganisms, and risk factors for infections within 30 days.

Identification and antimicrobial susceptibility test of the bacteria isolated from post-operative urine and blood cultures were collected from the laboratory information management system (Glims, MIPS, Gent, Belgium). Identification of the bacteria was performed by MALDI-TOF MS (MALDI Biotyper, Bruker, Daltonics, Bremen, Germany). Coagulase-negative staphylococci and viridans streptococci were considered not clinically relevant and were excluded from the analysis. Antimicrobial susceptibility testing was performed with Phoenix (BD Bioscience, Erembodegem, Belgium) and E-test (BioMérieux, AB Biodisk, Solna, Sweden) in accordance with European Committee on Antimicrobial susceptibility testing (EUCAST) methodology. Minimal inhibitory concentration (MIC) values were interpreted and categorized (S, I, and R) according to EUCAST clinical breakpoint table version 7.1. Antimicrobial susceptibility test results for Enterobacteriaceae included amoxicillin (AMX), amoxicillin-clavulanic acid (AMC), piperacillin-tazobactam (PTZ), ceftriaxone (CRO), ceftazidime (CAZ), ciprofloxacin (CIP), trimethoprimsulfamethoxazole (SXT), meropenem (MEM), and gentamicin $(\mathrm{CN})$. Susceptibility to cefazolin was not assessed since antimicrobial susceptibility EUCAST breakpoints for cefazolin for Enterobacteriaceae do not exist. Susceptibility testing for enterococci included AMX and vancomycin (VAN). Susceptibility testing for staphylococci included cefoxitin (screening test for methicillin susceptibility), and for Pseudomonas aeruginosa CIP, CAZ, CN, and MEM. In the analysis of susceptibility profiles we selected the first isolate per species per patient to avoid bias due to multiple testing. In case more than one bacterial species was isolated from a culture, all uropathogens with a unique resistance pattern were included.

Statistical analysis was performed using SPSS 22.0. Continuous variables were analyzed using students t-test in case of normal distribution, and non-parametric tests in case of non-normal distribution. Categorical variables were analyzed using Chi-Square or Fisher's exact test. We performed multivariate logistic regression analysis to test for risk factors for infection. Prognostic factors associated with a $p$-value of $<0.20$ in univariate analysis were included in multivariate analysis. A 2-sided $p$-value of $\leq 0.05$ was considered statistically significant.

The regional institutional review board approved this study and waived the requirement to obtain informed consent.

\section{Results}

\section{Patient characteristics}

Of the 200 patients who underwent radical cystectomy, 36 did not receive surgery at our institution. Of the 164 remaining patients, 17 patients had an active infection at time of surgery and were excluded. The remaining 147 patients were included in our study. A total of 120 patients underwent IC diversion (82\%), while $27(18 \%)$ received a NB. Patients that received a NB were younger than those who received an IC diversion (mean age 57.9 [SD 9.55] vs 67.0 [SD 10.1] years, respectively; $p=0.004)$ and had a different distribution of ASA scores (1 [IQR 1-1] vs 1 [IQR 1-2]; $p=0.047)$. Table 2 shows the demographic and clinical characteristics of the included patients.

Table 1 Definitions used for post-operative infectious complications

\begin{tabular}{ll}
\hline $\begin{array}{l}\text { Complicated urinary tract } \\
\text { infection }\end{array}$ & $\begin{array}{l}\text { Temperature } \geq 38.5^{\circ} \mathrm{C} \text { and a positive urine culture }\left(\geq 10^{5} \mathrm{CFU} / \mathrm{ml} \text { and leucocytes in gram stain) without a positive }\right. \\
\text { blood culture or SSI } \\
\text { Surgical site infections } \\
\text { Bacteremia }\end{array} \quad$ Temperature $\geq 38.5^{\circ} \mathrm{C}$ and a positive blood culture with or without a positive urine culture \\
\hline
\end{tabular}


Table 2 Demographic and clinical characteristics of the 147 included patients

\begin{tabular}{|c|c|}
\hline Variable & $n(\%)$ or mean $\pm S D$ \\
\hline Sex, males & $112(76.2)$ \\
\hline Age, years & $65.4(10.6)$ \\
\hline $\mathrm{BMI}, \mathrm{kg} / \mathrm{m}^{2}$ & $26.4(4.1)$ \\
\hline (Previous) smoker & $68(46.3)$ \\
\hline Hypertension & $79(53.7)$ \\
\hline Diabetes mellitus & $16(10.9)$ \\
\hline Nephrostomy catheter & $14(10.1)$ \\
\hline \multicolumn{2}{|l|}{ ASA class } \\
\hline ASA class 1 & $20(13.6)$ \\
\hline ASA class 2 & $91(61.9)$ \\
\hline ASA class 3 & $36(24.5)$ \\
\hline \multicolumn{2}{|l|}{ Indication } \\
\hline Bladder cancer & $123(81.6)$ \\
\hline Stage $T \geq T 2$ & $73(59.3)$ \\
\hline Other malignancy & $13(8.8)$ \\
\hline Functional & $8(5.4)$ \\
\hline Other & $3(2.0)$ \\
\hline Colonization with MDRO & $7(4.8)$ \\
\hline Guideline-accordant prophylaxis & $116(78.9)$ \\
\hline Correct choice & $134(91.2)$ \\
\hline Correct dose & $124(92.5)$ \\
\hline Administration $<60$ min prior to incision & $142(91.2)$ \\
\hline \multicolumn{2}{|l|}{ Type of diversion } \\
\hline Ileal conduit & $120(81.6)$ \\
\hline Hautmann neobladder & $27(18.4)$ \\
\hline Duration surgery, minutes & $216(75)$ \\
\hline Blood loss, ml & $1031(700)$ \\
\hline
\end{tabular}

Abbreviations: ASA American Society of Anesthesiologists, BMI body mass index, IQR interquartile range, $M D R O$ multidrug resistant microorganism, $S D$ standard deviation

\section{Incidence of post-operative infections}

During the 30-day post-operative period 82 post-operative infections occurred in 69 of 147 patients (46.9\%) (Fig. 1). We found 27 episodes of bacteremia (in 27 patients [32.9\%]), 43 complicated UTIs (in 41 patients [52.4\%], and 12 SSIs (in 11 patients [14.6\%]). Twelve patients had more than one infection during the study period. The incidence of post-operative infections peaked on day 4-5 and day 8-10 post-operatively (Fig. 1). The highest incidence of bacteremia was on day 7-10, whereas SSIs only occurred from day 5 onwards and were evenly distributed during the 30-day follow-up period.

\section{Bacteremia}

Twenty-seven of 147 patients had bacteremia during the 30-day post-operative period (18.4\%). Of 27 patients with positive blood cultures, in 17 patients both the blood culture and urine culture contained identical bacteria, in 7 patients no urine cultures were performed, and in 3 patients the bacteria found in the blood culture differed from the bacteria found in the urine culture. The blood cultures performed grew a total of 28 bacteria (Table 3). Enterobacteriaceae represented the largest group (67.9\%), followed by enterococci (18\%), S. aureus (11\%) and B. fragilis (4\%). In one patient, the blood culture contained both Klebsiella pneumoniae and Proteus mirabilis. Susceptibility profiles of the Enterobacteriaceae plus $P$. aeruginosa are shown in Fig. 2a. Enterococci were susceptible to AMX in $80 \%$ of isolates and susceptibility to VAN was $100 \%$. All S. aureus isolates were methicillin susceptible.

\section{Complicated UTI}

Forty-one of 147 patients had at least one episode of cUTI during the 30-day post-operative period (27.9\%). The 43 urine cultures of these 41 patients grew 66 micro-organisms (range of cultured bacteria within 1 culture: $1-3)$, of which $41(62.1 \%)$ were Enterobacteriaceae (Table 3). The susceptibility profiles of the

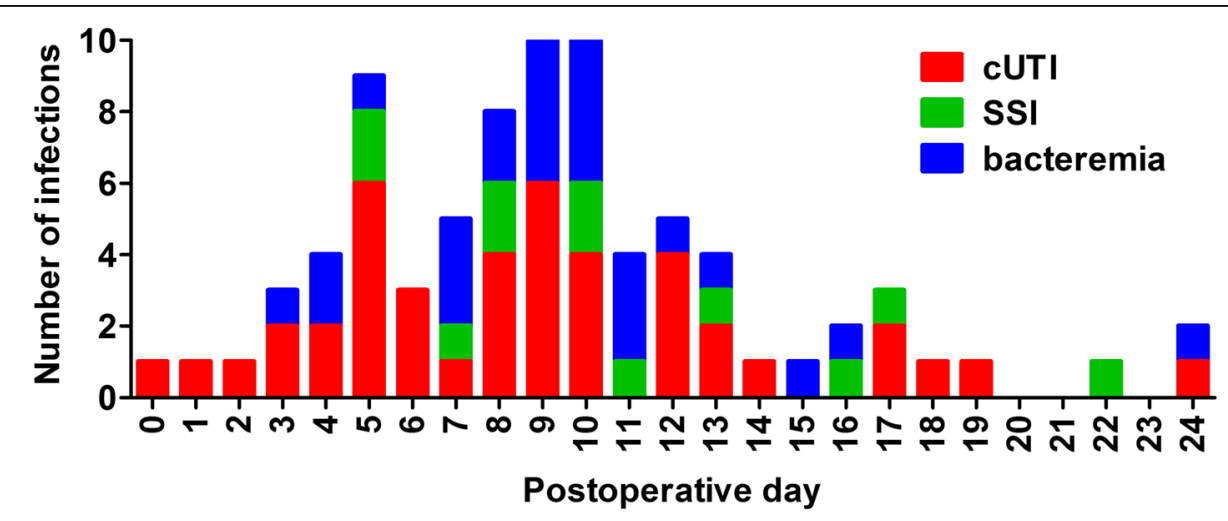

Fig. 1 Incidence and distribution in time of the post-operative infections 
Table 3 Urine and blood culture isolates from patients with post-operative infections

\begin{tabular}{|c|c|c|}
\hline Bacteria & Urine culture isolates, $n(\%)$ & Blood culture isolates, $n(\%)$ \\
\hline Enterobacteriaceae & $41(62.1)$ & $19(67.9)$ \\
\hline Escherichia coli & $14(34.2)$ & $8(42.1)$ \\
\hline Klebsiella pneumoniae & $9(22.0)$ & $5(26.3)$ \\
\hline Enterobacter spp. & $13(31.7)$ & $4(21.0)$ \\
\hline Proteus mirabilis & $0(0)$ & $1(5.3)$ \\
\hline Serratia marcescens & $1(2.4)$ & $1(5.3)$ \\
\hline Morganella morganii & $1(2.4)$ & 0 \\
\hline Citrobacter spp. & $2(4.9)$ & 0 \\
\hline Hafnia alvei & $1(2.4)$ & 0 \\
\hline Pseudomonas aeruginosa & $3(4.6)$ & 0 \\
\hline Enterococci & $16(24.2)$ & $5(17.9)$ \\
\hline Staphylococcus aureus & $6(9.1)$ & $3(10.7)$ \\
\hline Bacteroides fragilis & 0 & $1(3.5)$ \\
\hline Total & $66(100)$ & $28(100)$ \\
\hline
\end{tabular}

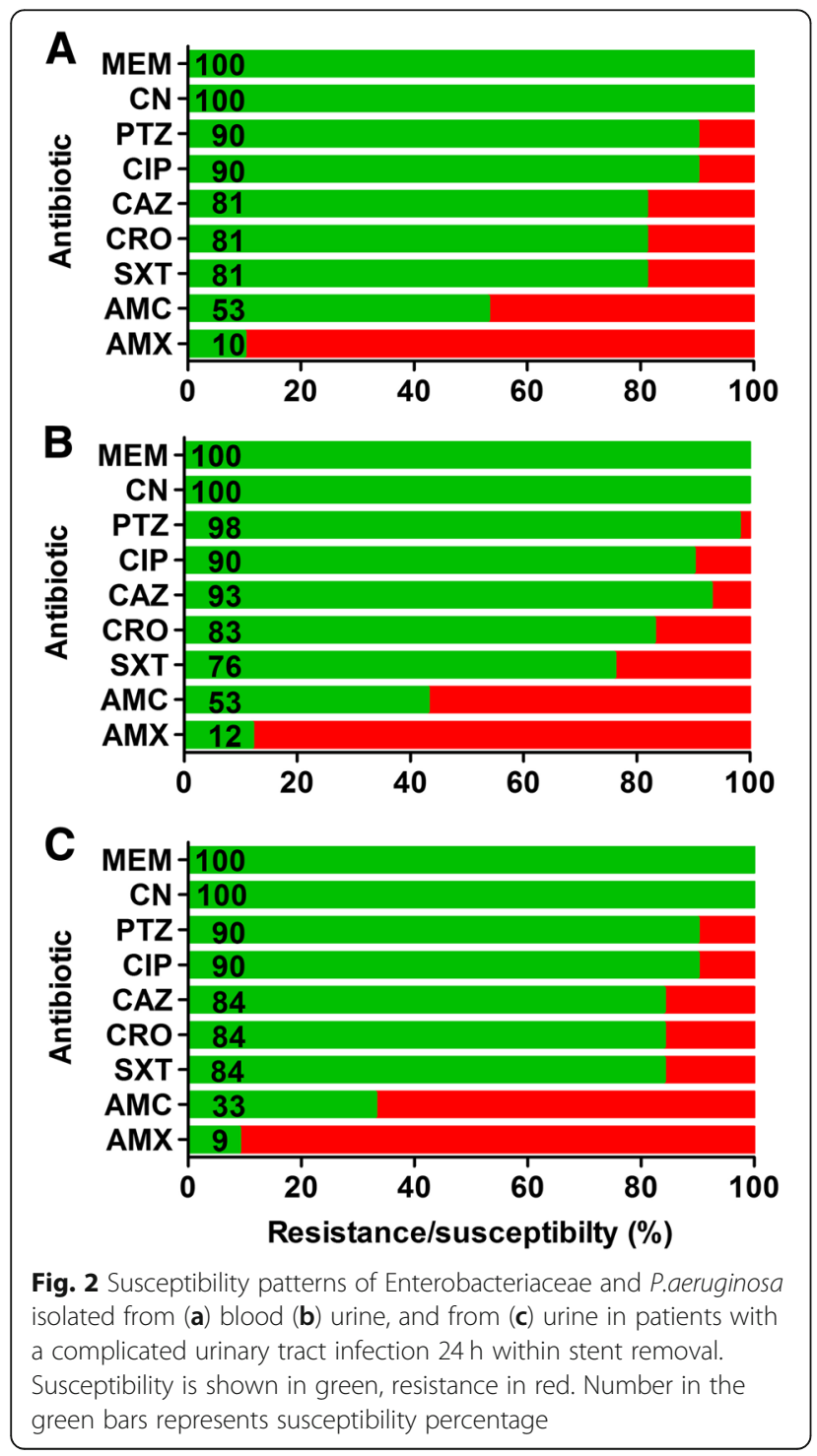

Enterobacteriaceae plus $P$. aeruginosa are shown in Fig. 2b. Enterococci were cultured in 16 (24.2\%) of 66 isolates and $75 \%$ of the isolates were susceptible to AMX and $100 \%$ to VAN. S. aureus formed $9.1 \%$ of cultured bacteria and $100 \%$ were methicillin susceptible.

\section{Infections after ureteral stent removal}

In the $24 \mathrm{~h}$ after ureteral stent removal, which was generally performed on post-operative day 7 through 10 (85.7\%), 7 patients developed a bacteremia. This was $25.9 \%$ of all patients with a bacteremia found in this study. Six of 7 blood cultures (85.7\%) grew Enterobacteriaceae, 1 blood culture grew B. fragilis (Table 4). In addition, 16 patients developed a complicated UTI within $24 \mathrm{~h}$ after ureteral stent removal. This was $37.2 \%$ of all episodes of cUTI found in this study. The 16 positive urine cultures grew a total of 24 micro-organisms (16 [66.7\%] Enterobacteriaceae, 5 [20.8\%] enterococci, 2 [8.3\%] were S. aureus and 1 [4.2\%] $P$. aeruginosa) (Table 4). Susceptibilities of the Enterobacteriaceae plus Pseudomonas from both urine and blood culture isolates are shown in Fig. 2c.

\section{Outcome and risk factors for post-operative infections}

Six patients $(4.1 \%)$ required surgical treatment, and 8 patients $(5.4 \%)$ were admitted to the ICU as a result of the infection. The median duration of admission was 14 days (IQR 5 days) and the 30-day mortality rate was $1.4 \%$ $(n=2)$, one of whom died of sepsis. In univariate analysis, type of diversion (NB) and MDRO colonization prior to surgery were associated with post-operative infections (Additional file 1: Table S1). Multivariate logistic regression analysis showed only $\mathrm{NB}$ to be associated with increased infections complications: odds ratio 4.1 (95\% confidence interval 1.6-10.5), $p=0.03$. 
Table 4 Urine and blood culture isolates within $24 \mathrm{~h}$ after ureteral stent removal

\begin{tabular}{|c|c|c|}
\hline Bacteria & Urine culture isolates, $n(\%)$ & Blood culture isolates, $n(\%)$ \\
\hline Enterobacteriaceae & $16(66.7)$ & $6(85.7)$ \\
\hline Escherichia coli & $4(25.0)$ & $3(50.0)$ \\
\hline Klebsiella pneumoniae & $2(12.5)$ & $1(16.7)$ \\
\hline Enterobacter spp. & $8(50.0)$ & $2(33.3)$ \\
\hline Proteus mirabilis & 0 & 0 \\
\hline Serratia marcescens & 0 & 0 \\
\hline Morganella morganii & $1(6.25)$ & 0 \\
\hline Citrobacter spp. & $1(6.25)$ & 0 \\
\hline Pseudomonas aeruginosa & $1(4.2)$ & 0 \\
\hline Enterococci & $5(20.8)$ & 0 \\
\hline Staphylococcus aureus & $2(8.3)$ & 0 \\
\hline Bacteroides fragilis & 0 & $1(14.3)$ \\
\hline Total & $24(100)$ & $7(100)$ \\
\hline
\end{tabular}

\section{Discussion}

This study showed a high incidence of post-operative infections after radical cystectomy and urinary diversion surgery. Post-operative infections peaked at day 4-5. Furthermore, stent removal (around day 7-10) was associated with the occurrence of bacteremia and cUTI. The cultured gram-negative bacteria in urine and blood samples largely showed an inferred resistance to the used antibiotic prophylaxis regimen consisting of cefazolin. These findings question the timing and choice of cefazolin, currently the most used agent, as surgical antibiotic prophylaxis in radical cystectomy [13-16].

The assessment of etiology and antibiotic susceptibility of post-operative infections characteristics in this study enabled us to broaden the spectrum of antibiotic prophylaxis for radical cystectomy in our hospital to piperacillin/tazobactam and also administer empirical antibiotic prophylaxis just before removal of the ureteral stents. A detailed analysis of the etiology and antibiotic susceptibility of urine and blood isolates does highlight the importance of selecting prophylaxis using the local resistance data. Therefore, this study is an illustrative example that a local guideline should be adapted to local epidemiology of resistance [12, 21]. Another strength of this study is the involvement of stakeholders, as recommended by The Appraisal of Guidelines for REsearch \& Evaluation (AGREE) Instrument, a methodological strategy for the development of guidelines [22]. As professional taking care of the target population, the urologist noticed the high incidence of post-operative infections and also hypothesized the relationship with stent removal. Subsequently, the collaboration with a clinical microbiologist and infectious disease specialist enabled the current study and the development of a new guideline. Importantly, the involvement of the prescribing professional, i.e. the urologist, leads to an increased confidence in and facilitates a better implementation of and adherence to a new guideline [22].

Several studies assessed the risk of early post-operative infections after radical cystectomy, showing high incidences but large differences in reported post-operative infection rates. [4-11] In our study, bacteremia was found in $18 \%$, SSI in $8.2 \%$ and cUTI in $28 \%$ of patients. Rates of early post-operative bacteremia, SSI and UTI (<30 days) in other studies varied between 5 to $17 \%, 8$ to $33 \%$ and 4 to $36 \%$, respectively. [5, 9-11] One explanation for the large differences in reported incidences of post-operative infections after radical cystectomy might be the different types of antibiotic prophylaxis regimens used in different studies. Pariser et al. showed that type of peri-operative antibiotic prophylaxis regimen might have significant effect on infection outcomes after radical cystectomy [7], which was also found in two other studies that analyzed post-operative infections within 90 days after UDS [14, 23]. A recent cohort study in 8351 patients from 353 hospitals in the United States showed that 579 unique peri-operative antibiotic prophylaxis regimens were used. Only $28 \%$ of patients received prophylaxis according to the guideline [14]. Also, the diagnosis of cUTI in patients with urinary diversion is complicated by its vague presentation and lack of a standardized definition in the literature. Even though in our study we only diagnosed cUTI when fever $\left(\geq 38.5^{\circ} \mathrm{C}\right)$ could not be explained by SSI or bacteremia (and urine culture showed $\geq 10^{5} \mathrm{CFU} / \mathrm{ml}$ bacteria), we are aware that we might have over reported the amount of cUTI in this study as fever and bacteriuria are common after UDS surgery.

This study particularly focused on the temporal relationship of post-operative infections and the removal of the ureteral stents. To the best of our knowledge, this is 
the first study that assessed the etiology and antimicrobial susceptibility of bacteria cultured from blood and urine within $24 \mathrm{~h}$ after stent removal. We found that $26 \%$ of all episodes of bacteremia and $37 \%$ of all episodes of cUTI occurred within $24 \mathrm{~h}$ after ureteral stent removal. Current guidelines do not recommend on the need and type of antibiotic prophylaxis during ureteral stent removal after UDS $[13,15,16]$. Most probably because the temporal relationship between infection and ureteral stent removal has not been investigated thoroughly, as we could only find two previous studies on this topic $[10,24]$. Recently, Werntz et al. analyzed the difference in post-operative infectious complications between 42 patients that received peri-operative and 42 patients that received peri-operative plus extended (30 days) antibiotic prophylaxis after UDS. [10] They found that $30 \%$ of patients that received peri-operative prophylaxis only developed a cUTI one day after ureteral stent removal compared to none of the patients in the group that received extended prophylaxis. In a retrospective study performed by Hashimoto et al., prophylactic administration of antibiotics just before stent removal significantly reduced the incidence of febrile events after removal of ureteral stents [24]. Given the high incidence of bacteremia and cUTI within $24 \mathrm{~h}$ after stent removal in our study, we believe that, based on the results of this study, antibiotic prophylaxis during ureteral stent removal might reduce post-operative infections after UDS. We encourage administration of a single dose of prophylaxis just before ureteral stent removal rather than extended post-operative antimicrobial prophylaxis, which was administered by Werntz et al. in their study, as this may lead to the development of antimicrobial resistance. $[13,16]$ However, due to the retrospective and single center design of our study, we feel these findings need to be confirmed in a future prospective randomised clinical trial.

This study has several limitations. The variables of interest were collected retrospectively, which brings along the risk of missing data. However, the outcome parameters are recorded in our EMR and laboratory systems as discrete variables that can be retrieved easily, reducing the risk. Whether a patient is colonized with MDRO was not systematically assessed prior to the operation in most patients, limiting the possibility to draw a conclusion on tailoring prophylaxis to carrier state or structural screening for carrier state of MDRO. Another limitation is that this is a single center study in a country with (relatively) low-level resistance of Enterobacteriaceae. Ideally, the effect of a change in antibiotic prophylaxis regimen should be examined in a robustly designed clinical follow-up study. A final limitation of the study is that the susceptibility of Enterobacteriaceae to cefazolin was not tested as EUCAST breakpoints for cefazolin do not exist. The susceptibility of
Enterobacteriaceae to cefazolin is roughly comparable to the susceptibility to AMX and second generation cephalosporins [25], implying that cefazolin does not cover the majority of Enterobacteriaceae that caused the post-operative infections found in this study.

\section{Conclusions}

A detailed analysis of the incidence, etiology, and timing of infections following radical cystectomy showed high incidence of post-operative infections after radical cystectomy and urinary diversion surgery. Notably, we identified a peak in bacteremia and complicated UTI immediately after stent removal. We believe that administration of antibiotic prophylaxis just before stent removal might help to reduce post-operative infections, although this needs to be confirmed in future robust clinical trials before (inter)national guidelines should be altered. Our study emphasizes that local guidelines should be adapted to local epidemiology of resistance, and that increased antimicrobial resistance necessitates a prophylactic regimen with increased gram-negative coverage in radical cystectomy.

\section{Additional file}

\section{Additional file 1: Table S1. Uni- and multivariate analysis of post-operative} infections after radical cystectomy. (DOCX $15 \mathrm{~kb}$ )

\section{Abbreviations}

AMC: amoxicillin-clavulanic acid; AMX: Amoxicillin; ASA: American Society of Anesthesiologists; BMI: Body mass index; CAZ: Ceftazidim; CCl: Charlson Comorbidity Index; CIP: Ciprofloxacin; CN: Gentamicin; CRO: Ceftriaxon; CUTI: Complicated urinary tract infection; EUCAST: European Committee on Antimicrobial susceptibility testing; IC: Ileal conduit; MALDI-TOF

MS: Matrix-assisted laser desorption-ionization-time-of-flight mass spectrometry; MDRO: Multidrug resistant organisms; MEM: Meropenem;

MIC: Minimal inhibitory concentration; NB: Neobladder;

PTZ: Piperacillin-tazobactam.; SSI: Surgical site infection;

SXT: Trimethoprim-sulfamethoxazole; UDS: Urinary diversion surgery; VAN: Vancomycin

Acknowledgements

Not applicable.

Funding

None.

\section{Availability of data and materials}

The datasets used and/or analysed during the current study are available from the corresponding author on reasonable request.

\section{Author's contributions}

All authors have read and approved the manuscript. EK: Protocol/project development; Data collection or management; Data analysis; Manuscript writing/editing AS: Data collection or management; Manuscript writing/ editing ST: Manuscript writing/editing TVdH: Data collection or management: Manuscript writing/editing MS: Protocol/project development; Manuscript writing/editing JtO: Protocol/project development; Data collection or management; Data analysis; Manuscript writing/editing.

Ethics approval and consent to participate

The regional institutional review board of the Radboudumc approved this study and waived the requirement to obtain informed consent. No 
administrative permissions were required to access the raw data from the Radboudumc

\section{Consent for publication}

Not applicable.

\section{Competing interests}

The authors declare that they have no competing interests.

\section{Publisher's Note}

Springer Nature remains neutral with regard to jurisdictional claims in published maps and institutional affiliations.

\section{Author details}

${ }^{1}$ Radboud center for infectious diseases, Radboud university medical center, P.O. Box 9101, 6500, HB, Nijmegen, the Netherlands. ${ }^{2}$ Department of medical microbiology, Radboud university medical center, P.O. Box 9101, 6500, HB, Nijmegen, the Netherlands. ${ }^{3}$ Department of internal medicine, Radboud university medical center, Nijmegen, the Netherlands. ${ }^{4}$ Department of urology, Radboud university medical center, Nijmegen, the Netherlands.

Received: 19 November 2018 Accepted: 21 March 2019

Published online: 03 April 2019

\section{References}

1. van Hemelrijck M, Thorstenson A, Smith P, Adolfsson J, Akre O. Risk of inhospital complications after radical cystectomy for urinary bladder carcinoma: population-based follow-up study of 7608 patients. BJU Int. 2013;112(8):1113-20

2. Chang SS, Cookson MS, Baumgartner RG, Wells N, Smith JA Jr. Analysis of early complications after radical cystectomy: results of a collaborative care pathway. J Urol. 2002;167(5):2012-6.

3. Nieuwenhuijzen JA, de Vries RR, Bex A, van der Poel HG, Meinhardt W, Antonini $\mathrm{N}$, et al. Urinary diversions after cystectomy: the association of clinical factors, complications and functional results of four different diversions. Eur Urol. 2008:53(4):834-42 discussion 42-4.

4. Takeyama K, Matsukawa M, Kunishima Y, Takahashi S, Hotta H, Nishiyama $\mathrm{N}$, et al. Incidence of and risk factors for surgical site infection in patients with radical cystectomy with urinary diversion. J Infect Chemother. 2005;11(4):177-81.

5. Kim KH, Yoon HS, Yoon H, Chung WS, Sim BS, Lee DH. Febrile urinary tract infection after radical cystectomy and Ileal Neobladder in patients with bladder Cancer. J Korean Med Sci. 2016;31(7):1100-4.

6. Gondo T, Ohno Y, Nakashima J, Hashimoto T, Takizawa I, Tanaka A, et al. Factors predicting incisional surgical site infection in patients undergoing open radical cystectomy for bladder cancer. Int J Clin Oncol. 2014:19(5):935-9.

7. Pariser JJ, Anderson BB, Pearce SM, Han Z, Rodriguez JA 3rd, Landon E, et al. The effect of broader, directed antimicrobial prophylaxis including fungal coverage on perioperative infectious complications after radical cystectomy. Urol Oncol. 2016;34(3):121 e9-14.

8. Goldberg H, Shenhar C, Tamir H, Mano R, Baniel J, Margel D, et al. Predictors of surgical site infection after radical cystectomy: should we enhance surgical antibiotic prophylaxis? World J Urol. 2018.

9. Kaczmarek K, Leminski A, Bancarz A, Zakrzewska A, Slojewski M. Postoperative infections among patients undergoing radical cystectomy at a tertiary center. Surg Infect. 2018;19(4):451-8.

10. Werntz RP, Martinez-Acevedo A, Amadi H, Kopp R, La Rochelle J, Koppie T, et al. Prophylactic antibiotics following radical cystectomy reduces urinary tract infections and readmission for sepsis from a urinary source. Urol Oncol. 2018;36(5):238 e1-5

11. Parker WP, Tollefson MK, Heins CN, Hanson KT, Habermann EB, Zaid HB, et al. Characterization of perioperative infection risk among patients undergoing radical cystectomy: results from the national surgical quality improvement program. Urol Oncol. 2016;34(12):532 e13-9.

12. Cai T, Bonkat G, Tandogdu Z, Bartoletti R, Wagenlehner FME, Grabe M, et al. How to use antimicrobial prophylaxis in urological procedures. Eur Urol Focus. 2016;2(4):348-50.

13. Perioperative antibiotic prophylaxis Dutch Working Party on Antibiotic Policy (SWAB). 2017.
14. Krasnow RE, Mossanen M, Koo S, Kubiak DW, Preston MA, Chung Bl, et al. Prophylactic antibiotics and postoperative complications of radical cystectomy: a population based analysis in the United States. J Urol. 2017.

15. Bonkat G, Pickard R, Bartoletti R, Bruyère F, Geerlings SE, Wagenlehner F, et al. EUA guideline on urological infections. Retrieved from: http:// uroweborg/guideline/urological-infections/ Access date 08-01-2018.

16. JS W, CJ B, RR D, BK H, MS P, AJ S. AUA best practices statements urologic surgery antimicrobial prophylaxis. 2012.

17. van Kasteren ME, Gyssens IC, Kullberg BJ, Bruining HA, Stobberingh EE, Goris RJ. Optimizing antibiotics policy in the Netherlands. V. SWAB guidelines for perioperative antibiotic prophylaxis. Foundation antibiotics policy team. Ned Tijdschr Geneeskd. 2000;144(43):2049-55.

18. Szary NM, Al-Kawas FH. Complications of endoscopic retrograde cholangiopancreatography: how to avoid and manage them. Gastroenterol Hepatol (N Y). 2013;9(8):496-504

19. Weber WP, Mujagic E, Zwahlen M, Bundi M, Hoffmann H, Soysal SD, et al. Timing of surgical antimicrobial prophylaxis: a phase 3 randomised controlled trial. Lancet Infect Dis. 2017;17(6):605-14.

20. Mangram AJ, Horan TC, Pearson ML, Silver LC, Jarvis WR. Guideline for prevention of surgical site infection, 1999. Centers for Disease Control and Prevention (CDC) hospital infection control practices advisory committee. Am J Infect Control. 1999:27(2):97-132 quiz 3-4; discussion 96.

21. Schuts EC, Hulscher M, Mouton JW, Verduin CM, Stuart J, Overdiek H, et al. Current evidence on hospital antimicrobial stewardship objectives: a systematic review and meta-analysis. Lancet Infect Dis. 2016;16(7):847-56.

22. AGREE Next Steps Consortium (2009). The AGREE II instrument [electronic version]. Retrieved December, 31, 2017, from http://www.agreetrust.org.

23. Shigemura K, Tanaka K, Matsumoto M, Nakano Y, Shirakawa T, Miyata M, et al. Post-operative infection and prophylactic antibiotic administration after radical cystectomy with orthotopic neobladder urinary diversion. J Infect Chemother. 2012;18(4):479-84.

24. Hashimoto J, Takahashi S, Kurimura Y, Takeyama K, Kunishima Y, Tsukamoto T. Clinical relevance of single administration of prophylactic antimicrobial agents against febrile events after removal of ureteral stents for patients with urinary diversion or reconstruction. Int J Urol. 2010;17(2):163-6.

25. Livermore DM. Beta-lactamases in laboratory and clinical resistance. Clin Microbiol Rev. 1995:8(4):557-84.
Ready to submit your research? Choose BMC and benefit from:
- fast, convenient online submission
- thorough peer review by experienced researchers in your field
- rapid publication on acceptance
- support for research data, including large and complex data types
- gold Open Access which fosters wider collaboration and increased citations
- maximum visibility for your research: over $100 \mathrm{M}$ website views per year
At BMC, research is always in progress.
Learn more biomedcentral.com/submissions 